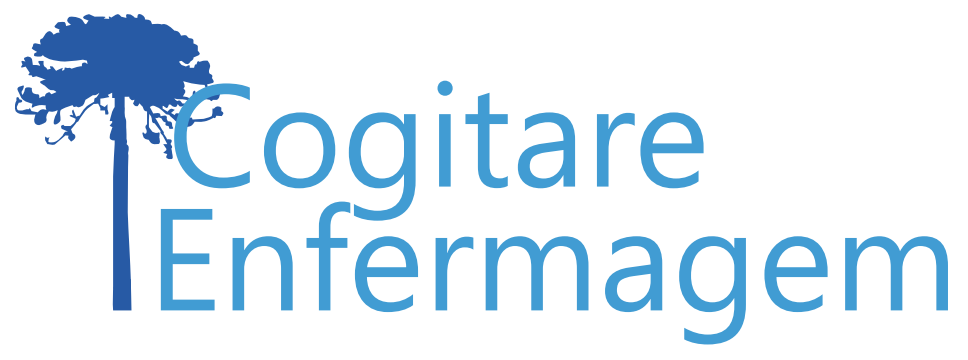

\title{
CONSTRUÇÃO E VALIDAÇÃO DE APLICATIVO DIGITAL PARA ENSINO DE INSTRUMENTAÇÃO CIRÚRGICA
}

Francisco Gilberto Fernandes Pereira1, Driele Jéssica Leite da Rocha², Geórgia Alcântara Alencar Melo $^{3}$, Rhaylla Maria Pio Leal Jaques ${ }^{4}$, Laura Maria Feitosa Formiga ${ }^{5}$

\section{RESUMO}

Objetivo: construir e validar aplicativo sobre ensino de instrumentação cirúrgica básica para acadêmicos de enfermagem.

Método: estudo metodológico, desenvolvido entre maio e setembro de 2016, em universidade pública do Piauí, Brasil. Seguiram-se as etapas de análise, desenho, desenvolvimento e avaliação, com base no referencial de Galvis-Panqueva. A validação de conteúdo e aparência foi realizada com 22 profissionais, e a avaliação com o público-alvo correspondeu a 60 acadêmicos de enfermagem. Resultados: 0 aplicativo foi nomeado de Instrumentais Cirúrgicos e está hospedado na plataforma Google play, disponível para uso no sistema operacional Android. Apresentou Indice de Validade de Conteúdo global de 0,9 quanto ao conteúdo e 0,8 quanto à aparência. O público-alvo avaliou o aplicativo como excelente, quanto aos itens de motivação, estilo e conteúdo, com média de $76,3 \%$.

Conclusão: o aplicativo mostrou-se válido para utilização como ferramenta auxiliar de ensino sobre o assunto instrumentação cirúrgica.

DESCRITORES: Tecnologia educacional; Educação em enfermagem; Instrumentos cirúrgicos; Enfermagem de centro cirúrgico; Enfermagem.

COMO REFERENCIAR ESTE ARTIGO:

Pereira FGF, Rocha DJL, Melo GAA, Jaques RMPL, Formiga LMF. Construção e validação de aplicativo digital para ensino de instrumentação cirúrgica. Cogitare enferm. [Internet]. 2019 [acesso em "colocar data de acesso, dia, mês abreviado e ano"]; 24. Disponível em: http://dx.doi.org/10.5380/ce.v24i0.58334.

Este obra está licenciado com uma Licença Creative Commons Atribuição 4.0 Internacional.

${ }^{1}$ Enfermeiro. Mestre em Enfermagem. Docente da Universidade Federal do Piauí. Picos, PI, Brasil. ()

Enfermeira. Universidade Federal do Piauí. Picos, PI, Brasil. $\bigcirc$

${ }^{3}$ Enfermeira. Mestre em Enfermagem. Universidade Federal do Ceará. Fortaleza, CE, Brasil. $\bigcirc$

${ }^{4}$ Enfermeira. Universidade Federal do Piauí. Picos, PI, Brasil. 9

${ }^{5}$ Enfermeira. Mestre em Farmacologia. Docente da Universidade Federal do Piauí. Picos, PI, Brasil. 9 


\title{
BUILDING AND VALIDATING A DIGITAL APPLICATION FOR THE TEACHING OF SURGICAL INSTRUMENTATION
}

\begin{abstract}
Objective: To build and validate an application on teaching of basic surgical instrumentation to nursing students.

Method: Methodological study conducted between May and September of 2016, at a public university in Piauí, Brazil. Analysis, design, development and evaluation stages were conducted based on the Galvis-Panqueva benchmark. Content and appearance validation was performed by 22 judges, and 60 nursing students selected by convenience sampling were the target audience that evaluated the instrument.

Results: The application was named Surgical Instruments and is hosted on the Googleplay platform, available for use on the Android operating system. It had a global Content Validity Index of 0.9 for content and 0.8 for appearance. The target audience rated the application as excellent for motivation, style and content items, averaging $76.3 \%$.

Conclusion: The application has proved to be valid for use as an auxiliary teaching tool on the subject surgical instrumentation.
\end{abstract}

DESCRIPTORS: Educational technology; Nursing education; Surgical instruments; Surgical center nursing; Nursing.

\section{CONSTRUCCIÓN Y VALIDACIÓN DE APLICACIÓN DIGITAL PARA ENSEÑANZA DE INSTRUMENTACIÓN QUIIRÚRGICA}

\section{RESUMEN}

Objetivo: construir y validar aplicación asociada a la enseñanza de instrumentación quirúrgica básica para académicos de enfermería.

Método: estudio metodológico, desarrollado entre mayo y septiembre de 2016, en universidad pública de Piauí, Brasil. Se realizaron las etapas de análisis, proyecto, desarrollo y evaluación, con base en el referencial de Galvis-Panqueva. La validación de contenido y apariencia se hizo con 22 profesionales, y la evaluación con el público objetivo correspondió a 60 académicos de enfermería.

Resultados: el nombre de la aplicación es "Instrumentais Cirúrgicos" y ella está en la plataforma Google play, disponible para uso en el sistema operacional Android. El Índice de Validad de Contenido global fue de 0,9 cuanto al contenido y 0,8 cuanto a la apariencia. El público objetivo evaluó la aplicación como excelente, cuanto a los criterios motivación, estilo y contenido, con promedio de $76,3 \%$.

Conclusión: la aplicación fue eficaz para utilización como herramienta auxiliar de enseñanza acerca del asunto instrumentación quirúrgica.

DESCRIPTORES: Tecnología educacional; Educación en enfermería; Instrumentos quirúrgicos; Enfermería de centro quirúrgico; Enfermería. 
O uso das Tecnologias da Informação e Comunicação (TIC), como ferramenta auxiliar no processo de ensino-aprendizagem, tem se mostrado eficaz na construção do conhecimento e no desenvolvimento de habilidades manuais em diversas áreas e níveis de ensino(1).

A Enfermagem tem colaborado para a produção dessas tecnologias nas áreas educacionais, com dispositivos para mediação de metodologias de ensinar e aprender, utilizados entre educadores e educandos nos vários processos de educação, como formal-acadêmica e formal-continuada; assistenciais, com equipamentos para mediação de processos de cuidar aplicados por profissionais com clientes-usuários dos sistemas de saúde na atenção primária, secundária e terciária; e gerenciais, com artefatos para mediação de processos da gestão, utilizadas por profissionais em serviços e unidades dos distintos sistemas de saúde ${ }^{(2)}$.

As tecnologias educacionais se destacam em relação às demais, devido à própria situação cultural vivenciada na contemporaneidade, em que a formação profissional exige, além do conhecimento teórico, pensamento crítico, reflexivo, versátil, além de possibilitar transformar a realidade social a partir dos recursos disponíveis ${ }^{(3)}$.

O contato com a tecnologia está presente nas diversas áreas da Enfermagem cotidianamente. No entanto, algumas têm demonstrado ganhos mais eminentes que outras, como no caso do cuidado assistencial perioperatório. Observa-se a evolução dos procedimentos cirúrgicos, com técnicas minimamente invasivas, cirurgias robóticas, que usam maquinário extremamente sofisticado para garantir maior assertividade cirúrgica e menor risco de infecção(4).

Percebe-se a necessidade de inserção de novos materiais para o processo de ensinoaprendizagem na formação do enfermeiro na área de instrumentação cirúrgica, pois durante a graduação, os discentes não se sentem aptos a realizar procedimentos voltados ao ato cirúrgico, bem como a manipular instrumentais que auxiliem a equipe cirúrgica para o exercício desta função.

A escolha por esta temática justifica-se devido às dúvidas, dificuldades e à ausência de ferramenta educativa do tipo software móvel, como recurso adicional para o ensino na graduação, bem como pela necessidade de conhecimento sobre o tema, uma vez que os acadêmicos de enfermagem referem lacunas nesse aspecto, pois não estão familiarizados com a rotina do centro cirúrgico.

Diante do exposto, há necessidade de tornar os acadêmicos aptos a desenvolverem a atividade de instrumentação cirúrgica com segurança e qualidade. Logo, urge promover novas estratégias de aprendizagem acerca da instrumentação cirúrgica, a fim de colaborar para o desenvolvimento profissional, tendo a tecnologia como aliada e recurso promissor.

Pretendeu-se com este estudo, por meio de aplicativo, expandir os meios para o aprendizado em instrumentais cirúrgicos, para facilitar e despertar a curiosidade e a atenção de discentes. Assim, objetivou-se construir e validar aplicativo sobre o ensino de instrumentação cirúrgica básica para acadêmicos de enfermagem.

\section{MÉTODO}

Estudo metodológico, em que se propôs a criação de aplicativo para o ensino de instrumentação cirúrgica básica para acadêmicos de enfermagem.

O período do estudo foi entre maio e setembro de 2016. As etapas para operacionalização da pesquisa ocorreram de acordo com referencial metodológico de 
Galvis-Panqueva, devido à coesão com os objetivos da pesquisa. Assim, as fases desta pesquisa foram: construção (análise, design e desenvolvimento) e validação (avaliação)(5).

$\mathrm{Na}$ etapa de análise, definiram-se tema, objetivos, público-alvo, conteúdo a ser criado e tecnologia de infraestrutura. O tema instrumentação cirúrgica foi escolhido em virtude da demanda apresentada quanto às dificuldades que os alunos relataram na memorização de alguns instrumentais da caixa básica de cirurgias gerais e respectivas funções. Şeguidamente, realizou-se revisão da literatura especializada para definir o conteúdo do aplicativo.

Quanto ao desenho, foram estabelecidos o design instrucional, a estrutura de navegação, neste caso sob a forma de aplicativo, com download gratuito para plataforma Android, e a interface, para garantir interação, autonomia e facilidade em todos os comandos. O desenvolvimento, passo efetivo de execução, foi realizado por um engenheiro de software, com a supervisão dos pesquisadores.

Foram realizadas as validações de conteúdo e aparência, sendo a primeira por 11 enfermeiras, e a segunda, por 11 profissionais de tecnologia de informação, os quais foram identificados a partir da pesquisa na Plataforma Lattes, e contatados por e-mail. Optou-se pela formação de número ímpar de especialistas em cada área, seguindo a recomendação de outro estudo com abordagem similar(6).

Os critérios de inclusão foram: atingir três pontos de acordo com os seguintes itens: para enfermeiros - doutorado em enfermagem (4 pontos), professores com mestrado em educação em enfermagem (3 pontos), desenvolvimento de tecnologias em enfermagem educação (2 pontos), professor de enfermagem (1 ponto); e para profissionais de tecnologia da informação - doutorado em informática e tendo desenvolvido software (4 pontos), mestrado em informática e tendo desenvolvido software (3 pontos), graduado em informática e tendo desenvolvido software (2 pontos), formado em área de tecnologia informática (1 ponto).

Destaca-se que, em meio ao universo de enfermeiros e profissionais de tecnologia da informação que contemplam esses quesitos, os 11 primeiros juízes de cada área que confirmaram a participação no estudo, formaram o grupo de especialistas para a etapa de validação.

Para que os juízes pudessem realizar a avaliação, foi enviado o link para download do aplicativo e um formulário de avaliação com questões relativas à adequação da proposta, objetivos, organização, layout, escrita, aparência e motivação para material educacional.

As respostas foram apresentadas na escala Likert de: 1 (discordo) a 5 (concordo totalmente). Após receber os formulários, as respostas foram transferidas para uma base de dados, no Statistical Package for the Social Scienses (SPSS), e organizados em tabelas, com demonstração de frequência absoluta e medição de Índice de Validade de Conteúdo (IVC), considerando como item aprovado quando maior que 0,78, de acordo com a literatura para estudos de validação com mais de seis especialistas ${ }^{(7)}$. Ao final, foram realizados ajustes recomendados e disponibilizada a versão final.

Para validação junto ao público-alvo, foram selecionados por conveniência 60 acadêmicos de enfermagem que atendessem aos critérios de inclusão: estar cursando a disciplina de Enfermagem nas Cirurgias e Emergências de uma instituição pública de ensino superior brasileira e possuir aparelho celular operando com sistema Android. Durante a pesquisa, não houve perdas amostrais.

A pesquisa foi aplicada entre aqueles alunos que estavam presentes na sala de aula nos momentos da coleta de dados, a qual foi dividida em dois momentos. Primeiro, apresentação do aplicativo e recomendação de utilização por uma semana; e, segundo, após uma semana, responder a um questionário estruturado com 11 questões de múltipla escolha, nas quais a tecnologia foi abordada em três aspectos: motivação, estilo e conteúdo. 
Para avaliar a importância dos itens propostos, foi usada a escala de Likert, validada por meio de pré e pós-teste, que, neste caso, foram pontuados os itens: ruim, parcialmente bom, bom, muito bom e excelente. $O$ item foi considerado bem avaliado quando a concordância entre os respondentes foi maior ou igual a $70 \%$, nos itens muito bom e/ou excelente ${ }^{(6)}$.

Os dados foram organizados em tabelas e analisados por meio de estatística descritiva e inferencial, de acordo com a proposta da fase da pesquisa.

O estudo foi submetido ao Comitê de Ética da Universidade Federal do Ceará, aprovado conforme protocolo n. ${ }^{\circ} 983.129$.

\section{RESULTADOS}

Dos 11 especialistas do campo de Enfermagem que avaliaram o aplicativo, sete eram doutores e quatro mestres, e atuavam como professores em educação superior em Enfermagem. A Tabela 1 mostra a distribuição de avaliações realizadas por especialistas em enfermagem, com questões relativas à adequação da proposta, objetivos, organização, layout, escrita, aparência e motivação para material educativo.

Tabela 1 - Validação de conteúdo do aplicativo de acordo com a avaliação de enfermeiros especialistas. Picos, PI, Brasil, 2017

\begin{tabular}{|c|c|c|c|c|c|c|}
\hline Itens & D & DP & $\mathbf{C P}$ & $\mathbf{C}$ & $\mathbf{C T}$ & IVC \\
\hline $\begin{array}{l}\text { O aplicativo é apropriado para a proposta a que } \\
\text { se destina }\end{array}$ & - & - & - & 3 & 8 & 1 \\
\hline $\begin{array}{l}\text { O aplicativo facilita a aprendizagem dos } \\
\text { conceitos usados e suas aplicações }\end{array}$ & - & - & 4 & 2 & 5 & 0,6 \\
\hline O aplicativo fornece ajuda de forma completa & - & - & 2 & 6 & 3 & 0,8 \\
\hline Proporciona ajuda de forma não cansativa & - & - & - & 5 & 6 & 1 \\
\hline $\begin{array}{l}\text { O conteúdo do aplicativo corresponde ao } \\
\text { conteúdo presente em literaturas de enfermagem }\end{array}$ & - & - & - & - & 11 & 1 \\
\hline $\begin{array}{l}\text { O aplicativo é atrativo para estudantes de } \\
\text { enfermagem }\end{array}$ & - & - & - & 3 & 8 & 1 \\
\hline
\end{tabular}

O IVC global foi de 0,9 na avaliação dos juízes enfermeiros, e a maioria dos itens atingiu IVC superior a 0,78 , ou seja, nã̃o exigindo ajuste adicional. Os que atingiram a melhor pontuação foram: adequação da proposta; capacidade de fornecer ajuda de forma não cansativa; qualidade do conteúdo; e, atratividade, com IVC = 1 cada.

Quanto ao item relativo à facilitação da aprendizagem, o IVC alcançado foi de 0,6 . Foi pontuado pelos juízes que esse parâmetro somente poderia ser avaliado após realização de um estudo do tipo pré e pós-teste com grupos distintos.

Sobre os especialistas na área de tecnologia da informação, três eram mestres e oito possuíam apenas graduação. No entanto, tinham o requisito de desenvolvimento de um software, e o IVC global foi igual a 0,8. 
Tabela 2 - Validação de aparência do aplicativo, de acordo com a avaliação de especialistas em tecnologia da informação. Picos, PI, Brasil, 2017

\begin{tabular}{llccccc} 
Itens & D & DP & CP & C & CT & IVC \\
\hline O download do aplicativo é de fácil realização & - & - & - & 1 & 10 & 1 \\
\hline $\begin{array}{l}\text { A linguagem utilizada no aplicativo é de fácil } \\
\text { entendimento }\end{array}$ & - & - & 3 & 5 & 3 & 0,7 \\
\hline $\begin{array}{l}\text { Os recursos utilizados no aplicativo estão feitos } \\
\text { de forma correta }\end{array}$ & - & - & 4 & 5 & 2 & 0,6 \\
\hline $\begin{array}{l}\text { Os recursos utilizados no aplicativo são realizados } \\
\text { de forma abrangente }\end{array}$ & - & - & 2 & 5 & 4 & 0,8 \\
\hline A interface do aplicativo é atraente & - & - & - & 3 & 8 & 1 \\
\hline O aplicativo é fácil de manusear & & - & 2 & 7 & 2 & 0,8 \\
\hline $\begin{array}{l}\text { O aplicativo proporciona ajuda de forma não } \\
\text { cansativa }\end{array}$ & - & 3 & - & 3 & 5 & 0,7
\end{tabular}

*D: Discordo; DP: Discordo Parcialmente; CP: Concordo Parcialmente; C: Concordo; CT: Concordo Totalmente; IVC: Índice de Validade de Conteúdo.

De acordo com os dados apresentados na Tabela 2, os melhores itens classificados foram relativos ao download e à interface, os quais atingiram IVC $=1$ cada. Sinaliza-se que o item que avaliava a correta utilização dos recursos no aplicativo adquiriu IVC igual a 0,6, e os juízes recomendaram a implantação de novos atalhos e interatividade entre as telas, o que foi atendido pela equipe de desenvolvimento do aplicativo.

A avaliação do aplicativo foi realizada por 60 acadêmicos de enfermagem, sendo 45 mulheres e 15 homens, com idades entre 18 e 22 anos $( \pm 2,1)$. Conforme se observa na Tabela 3, os itens ruim e parcialmente ruim não pontuaram, apesar de constarem no instrumento de coleta.

Tabela 3 - Distribuição numérica e percentual da avaliação do aplicativo por estudantes, quanto à motivação, estilo e conteúdo. Picos, PI, Brasil, 2017 (continua)

\section{Variáveis}

Bom

Muito bom

Excelente

\section{Motivação}

\begin{tabular}{lcccccc}
\hline A interface do aplicativo é atrativa & 3 & $5 \%$ & 18 & $30 \%$ & 39 & $65 \%$ \\
\hline $\begin{array}{l}\text { É fácil baixar o aplicativo no celular no sistema } \\
\text { Android }\end{array}$ & 1 & $1,70 \%$ & 5 & $8,30 \%$ & 54 & $90 \%$ \\
\hline De forma geral, o aplicativo é de fácil manuseio & 10 & $16,70 \%$ & 13 & $21,70 \%$ & 37 & $61,60 \%$ \\
\hline O aplicativo fornece ajuda de forma efetiva & 5 & $8,30 \%$ & 5 & $8,30 \%$ & 50 & $83,40 \%$ \\
\hline
\end{tabular}

\section{Estilo}

\begin{tabular}{lcccccc}
\hline $\begin{array}{l}\text { A linguagem utilizada no aplicativo é de fácil } \\
\text { compreensão }\end{array}$ & 14 & $23,30 \%$ & 6 & $10 \%$ & 40 & $66,70 \%$ \\
\hline A interface do aplicativo é atrativa & 7 & $11,70 \%$ & 34 & $56,60 \%$ & 19 & $31,70 \%$ \\
\hline De forma geral, como você avalia o aplicativo? & 10 & $16,70 \%$ & 12 & $20 \%$ & 38 & $63,30 \%$ \\
\hline
\end{tabular}




\section{Conteúdo}

$\begin{array}{lllllll}\text { O aplicativo auxilia no processo de aprendizado } & 1 & 1,70 \% & 8 & 13,30 \% & 51 & 85 \%\end{array}$ dos instrumentais cirúrgicos

aplicativo otimiza o conhecimento acerca das

$60 \quad 100 \%$ nomenclaturas dos tempos cirúrgicos e seus

respectivos instrumentais

aplicativo aperfeiçoa a compreensão da utilização dos instrumentais cirúrgicos

O aplicativo auxilia no preparo para as avaliações práticas

No tocante à motivação, os subitens foram avaliados como excelente pela maioria dos estudantes, destacando-se que o download e a capacidade de fornecer ajuda efetiva foram aqueles mais bem avaliados, com 54 (90\%) e $50(83,4 \%)$ das respostas, respectivamente. $\mathrm{O}$ estilo foi pontuado em todos os subitens como bom, muito bom ou excelente, porém destaca-se que em relação à interface, a qual corresponde à aparência geral das telas do aplicativo, $34(56,6 \%)$ julgaram como muito bom, porém sinalizaram possibilidades de melhoria. Sobre o conteúdo, houve maior frequência de avaliação como excelente quanto à otimização do conhecimento sobre os instrumentais cirúrgicos e respectiva utilização, 60 (100\%) cada um.

\section{DISCUSSÃO}

O ingresso de tecnologias computacionais no processo educacional inova a relação ensino-aprendizagem, ao passo que se adapta às necessidades de modelos educacionais contemporâneos. A Enfermagem acompanha este processo e constata que as inovações tecnológicas aplicadas à prática clínica têm se mostrado vantajosas aos enfermeiros e também aos pacientes, apontando evidências de que essas habilidades, adequadamente utilizadas, podem ter impacto significativo nos cuidados prestados aos pacientes ${ }^{(6,8-9)}$.

O uso de aplicativos como ferramenta de ensino na área de saúde é bastante inovador, e apresenta-se como método capaz de gerar o interesse e a motivação em desejar aprender cada vez mais, haja vista que os aparelhos móveis, que hospedam esses aplicativos, são utilizados por profissionais de saúde na proporção de $45 \%$ a $85 \%$, sendo consultados mais que livros e revistas ${ }^{(10)}$.

Nesse cenário tecnológico, a expectativa dos acadêmicos em relação à inserção de tecnologias educacionais no aprendizado possibilita o acesso à informação em contextos externos à universidade. As informações são completas, principalmente em relação à aplicação prática, com disponibilização de materiais e aplicativos voltados para prática de determinados procedimentos ou técnicas ${ }^{(11)}$.

Desse modo, reconhece-se a importância desses profissionais se reinventarem, no que diz respeito à apropriação social das novas metodologias e tecnologias, na busca de proporcionar afinidade entre pessoas de diferentes culturas e conhecimentos, com objetivo de melhorar a aprendizagem ${ }^{(12)}$.

A utilização de um ambiente on-line apresenta vantagens para os professores, no que concernente à autonomia para criar propostas que possibilitem diferentes ferramentas de ensino, tornando esses espaços didáticos únicos. Neste sentido, o professor tem total liberdade para trabalhar com o aluno da melhor forma possível, otimizando o processo de ensino-aprendizagem, de acordo como princípio de que a tecnologia se torna mais desenvolvida, melhorada e adaptada na medida em que é utilizada ${ }^{(13)}$. 
No tocante aos recursos tecnológicos, os benefícios oferecidos à Enfermagem são perceptíveis; e a relação enfermagem/informática conquista espaço no meio acadêmico, pois computadores e dispositivos móveis aparecem de forma positiva no cenário da saúde educacional, causando impacto para sociedade. Logo, o profissional da saúde visualiza um novo papel a ser desempenhado, e como o uso dessas tecnologias resultará em um produto final positivo, ou seja, no sentido de beneficiar o paciente e racionalizar o trabalho, ${ }^{(14)}$ como é o caso do aplicativo ora desenvolvido.

Estudiosos da informática aplicada na área da Enfermagem mostram a relevância da utilização de recursos tecnológicos por docentes e acadêmicos de enfermagem, no sentido de tornar o uso das tecnologias cada vez mais inclusivas e interativas no processo de ensino-aprendizagem. Para tanto, o professor pode explorar a utilização de computadores, tablets, lousas digitais e smartphones para estimular alunos a praticarem situações que não vivenciaram ${ }^{(15)}$.

Diante disso, urge a importância da construção e disponibilização de mais recursos virtuais para o ensino na área da enfermagem, para que o aluno possa transpor para a realidade um nível mais elevado de conhecimento acerca da saúde e desenvolver habilidades cognitivas e técnicas.

Acrescenta-se que a execução do aplicativo Instrumentais Cirúrgicos apoiou-se no modelo desenvolvido ${ }^{(13)}$ ao considerar três dimensões: a presença cognitiva, a presença social e a presença de ensino. A primeira corresponde ao que os estudantes podem construir e confirmar, baseado em reflexão fundamentada e no discurso analítico. A presença social trata-se da habilidade que membros de uma comunidade têm de se projetarem social e emocionalmente, de acordo com o meio da comunicação que utilizam. Por fim, a presença de ensino é definida como uma junção da presença cognitiva e da presença social, ressaltando a direção, o designer e a facilitação dos mesmos, na perspectiva da realização dos resultados de aprendizagem pertinentes e que valham a pena ${ }^{(13)}$.

As informações circulantes de recursos oferecidos por computadores, pela Internet e outras redes de comunicação, mostram-se evidentemente mais ricas em formas e diversificadas em conteúdo do que as tradicionalmente existentes no ensino. Desse modo, conforme o objetivo, conteúdo e as características dos estudantes, vários recursos podem ser adotados pelo professor.

Ademais, estudos demonstram a eficiência da aplicação de recursos tecnológicos, como Moodle, aplicativos, redes sociais, fóruns e ambientes virtuais de aprendizagem, os quais propiciam a aquisição de conhecimento e habilidades cognitivas para efetuação de procedimentos de enfermagem, elevando a segurança e autoafirmação quanto à realização pelos estudantes. O uso dessas novas metodologias de ensino, através das tecnologias, acarreta resultados positivos que refletem na melhoria da prática dos enfermeiros ao oferecer mais confiança na realização do cuidado, e estabelecer o bem-estar de pacientes ${ }^{(16)}$.

É importante considerar que a utilização de aplicativos e outras modalidades computacionais não são necessariamente as melhores e únicas alternativas adequadas para o ensino, visto que o uso das tecnologias educacionais, mesmo que de forma adequada, não garante a eficácia da aprendizagem, que depende tanto da forma didática que a informação se apresentará, como do nível de interesse dos alunos em aprender ${ }^{(12)}$.

Neste sentido, em estudo sobre a obtenção de resultados positivos em relação ao uso de aplicativos educacionais por alunos, durante a realização de cálculos de medicamentos, mostrou a possibilidade da interação do aluno com a literatura especializada da área. É recomendado o uso dessa modalidade de ensino para estudantes de enfermagem, no intuito de facilitar o aprendizado, por ser um meio simples de ser utilizado, bem como pela acessibilidade ${ }^{(16)}$. Isto posto, é notória a identificação dos benefícios de aplicativos, visto que esta dispensa tempo por parte de alunos e professores e transmite segurança no momento dos resultados, desde que acrescentadas outras modalidades de ensinoaprendizagem. 
Outro estudo elucidou a construção de um aplicativo móvel que promoveu a interoperabilidade entre sistemas operacionais iOs, Android, Symbian, BlackBerrye webOs, consistindo em um aplicativo voltado para educação de profissionais de saúde e pacientes, utilizando-se do aplicativo framework, PhoneGap que permite a criação de aplicativos móbile usando padrões web. Também se utilizaram linguagens de programação Hyper Text Markup Language (HTML), Cascading Style Sheets (CSS) e JavaScript. Este aplicativo disponibiliza informações sobre vacinação, como composição da vacina, indicações, contraindicações e via de administração. Teve como resultado final excelente grau de usabilidade do software educacional(10).

Em outro estudo que trata de software educacional compatível com aparelhos que operam por tecnologias do tipo Android, a construção foi direcionada para estudantes de ensino superior e tem como objetivo ajudar estudantes sobre questões acerca do tema sinais vitais, incluindo temperatura, frequência respiratória, pressão arterial, frequência cardíaca, dor e Índice de Massa Corporal (IMC). Nesse estudo, os autores optaram por utilizar a metodologia de Galvis-Panqueva, ${ }^{(17)}$ por ser compatível com os objetivos da pesquisa. Encontraram-se semelhanças entre a construção deste software com o aplicativo desenvolvido para instrumentação çrúrgica trabalhada neste estudo, visto que há relativa similaridade na metodologia destes, e ambos se direcionam à mesma finalidade de complementar o modelo tradicional de ensino.

Convém destacar estudo de construção de software para ensino da técnica de cateterismo urinário de demora para acadêmicos de enfermagem, que utilizou modelo de aplicação tecnológica menos atual, que foi uma multimídia desenvolvida através dos

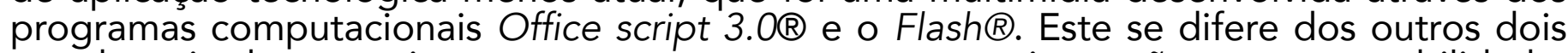
estudos citados anteriormente, por apresentar menor interação, menor mobilidade, necessidade de computador para acesso e baixa portabilidade. No entanto, ao realizar testes pré e pós-intervenção, foi comprovada a eficiência da multimídia na aquisição de conhecimento e habilidade ${ }^{(15)}$.

Portanto, é importante saber que as mudanças não devem partir somente do ponto de vista tecnológico, visto que este fato implica questões culturais, motiva tanto professores quanto alunos a repensarem os próprios papéis, a relação que há entre eles, nos conteúdos apropriados, nos quais resultam concretamente em plano de estruturação, ampliação de cursos e currículos, sistemas de avaliação, maneiras de ensinar e aprender, e metas a atingir. $O$ professor torna-se, portanto, tutor que garantirá que os sistemas de gestão de aprendizagem gerem impactos positivos no processo de ensino-aprendizagem ${ }^{(13)}$.

Pode-se apreender a partir desse contexto que a facilitação do desenvolvimento de pesquisas e trabalhos em redes de coparticipação pode acontecer devido às transformações sociais causadas pela influência direta da internet e da web, criando uma sociedade cuja informação pode ser construída e armazenada em diversos espaços e acessada pelos mais diversos usuários que estão separados pela distância geográfica(12).

Destarte, fica evidente que o desenvolvimento de aplicativos para o ensino de habilidades básicas em enfermagem é uma tendência contemporânea, e cabe às universidades, corpo de docentes e discentes, bem como aos enfermeiros que atuam na área assistencial, apropriarem-se dessas metodologias, no sentindo de fortalecer o desenvolvimento da profissão, investindo em cuidado mais seguro.

Destaca-se como limitação o fato de não ter sido realizada avaliação comparativa do aplicativo com outras metodologias de ensino acerca do mesmo tema, de modo que não se pode, ainda, afirmar efetividade quando comparada às demais formas de ensino utilizadas na atualidade.

CONCLUSÃO

O aplicativo demonstrou-se válido para utilização no cenário do ensino de 
instrumentação cirúrgica para estudantes de enfermagem e, diante disto, poderá servir como base para melhoria do modelo tradicional de ensino, pois poderá ser usado no cotidiano sem maiores dificuldades, já que pode ser consultado no modo off-line.

Ao analisar as dificuldades observadas sobre a instrumentação cirúrgica, quanto à técnica, terminologia e conceitos, é urgente que estratégias dinâmicas, interativas e inovadoras sejam adotadas no ensino de enfermagem, de modo que reduza a prática cristalizada de memorização estagnada. Vislumbra-se que este aplicativo trará segurança para o aluno, por se tratar de material educativo com grande disseminação de informações sobre a temática, e em proporção mais ampla, a tecnologia estudada também poderá ser estendida para os profissionais da área assistencial se assim desejarem utilizá-la.

\section{REFERÊNCIAS}

1. Silva AN, Santos AMG, Cortez EA, Cordeiro BC. Limites e possibilidades do ensino à distância (EaD) na educação permanente em saúde: revisão integrativa. Ciên. Saúde Colet. [Internet]. 2015 [acesso em 02 fev 2018]; 20(4). Disponível em: http://dx.doi.org/10.1590/1413-81232015204.17832013.

2. Fonseca LMM, Leite AM, Mello DF, Silva MAI, Lima RAG, Scochi CGS. Tecnologia educacional em saúde: contribuições para a enfermagem pediátrica e neonatal. Esc. Anna Nery. [Internet]. 2011 [acesso em 02 fev 2018]; 15(1). Disponível em: http://dx.doi.org/10.1590/S1414-81452011000100027.

3. Alarcon MFS, Prezotto KH. Evaluation of educational strategy, grounded on problem based learning on nursing undergraduate. Rev Rene. [Internet]. 2016 [acesso em 30 jan 2018]; 17(2). Disponível em: http:// www.redalyc.org/html/3240/324045343012/.

4. França AC, Camargo R. Desenvolvimento de um manipulador robótico para procedimentos cirúrgicos laparoscópicos: contribuições da engenharia mecânica. Rev. Ciênc Tecn. [Internet]. 2016 [acesso em 30 jan 2018]; 19(34). Disponível em: http://www.revista.unisal.br/sj/index.php/123/article/view/495.

5. Mendoza PB, Galvis AP. Ambientes virtuales de aprendizaje: una metodologia para su creación. Informática Educ. [Internet]. 1999 [acesso em 28 jan 2018]; 12(2). Disponível em: https://www. revistavirtualpro.com/biblioteca/ambientes-virtuales-de-aprendizaje-una-metodologia-para-su-creacion.

6. Holanda VR, Pinheiro AKB. Development of a hypermedia system for interactive e teaching of sexually transmitted diseases. Rev. enferm. UFPE on line. [Internet]. 2015 [acesso em 10 mar 2018]; 9(Suppl 2). Disponível em: https://periodicos.ufpe.br/revistas/revistaenfermagem/article/viewFile/10400/11165.

7. Moura IH, Silva AFR, Rocha AESH, Lima LHO, Moreira TMM, Silva ARV. Construction of educational materials for the prevention of metabolic syndrome in adolescents. Rev. Latino-Am. Enfermagem. [Internet]. 2017 [acesso em 10 mar 2018]; (25). Disponível em: http://dx.doi.org/10.1590/15188345.2024.2934.

8. Goyatá SLT, Chaves ECL, Andrade MBT, Pereira RJS, Brito TRP. Teaching the nursing process to undergraduates with the support of computer technology. Acta Paul Enferm. [Internet] 2012;25(2) [acesso em 13 fev 2018]. Disponível em: http://dx.doi.org/10.1590/S0103-21002012000200014.

9. Kobayashi RM, Leite MMJ. Technological competencies in cardiovascular nursing education. Rev Esc Enferm USP. [Internet]. 2015 [acesso em 04 fev 2018]; 49(6). Disponível em: http://dx.doi.org/10.1590/ S0080-623420150000600014.

10. Oliveira TR, Costa FMR. Development of mobile application for reference to vaccination In Brazil. J Health Inform. [Internet]. 2012 [acesso em 04 fev 2018]; 4(1). Disponível em: http://www.jhisbis.saude.ws/ojs-jhi/index.php/jhi-sbis/article/viewFile/161/109.

11. Frota NM, Barros LM, Costa AFA, Santos ZMSA, Caetano JA. Educational hypermedia on peripheral venipuncture: the perspective of students of nursing. Cogitare enferm. [Internet]. 2014 [acesso em 13 fev 2018]; 19(4). Disponível em: http://revistas.ufpr.br/cogitare/article/ viewFile/35384/23936. 
12. Marchiori LLM, Melo JJ, Melo WJ. Avaliação docente em relação às novas tecnologias para a didática e atenção no ensino superior. Avaliação. [Internet]. 2011 [acesso em 13 fev 2018]; 16(2). Disponível em: http://dx.doi.org/10.1590/S1414-40772011000200010.

13. Moreira JAM, Monteiro AM. O trabalho pedagógico em cenários presenciais e virtuais no ensino superior. Educ Form Tecn. [Internet]. 2010 [acesso em 10 fev 2018]; 3(2). Disponível em: https:// repositorio-aberto.up.pt/bitstream/10216/70533/2/87411.pdf.

14. Gomes AMT. Nursing care as a significant presence: an intersection between creativity and technology. Rev enferm UERJ. [Internet]. 2014 [acesso em 13 fev 2018]; 22(6). Disponível em: http:// dx.doi.org/10.12957/reuerj.2014.15783.

15. Lopes ACC, Ferreira AA, Fernandes JAL, Morita ABPS, Poveda VB, Souza AJS. Construction and evaluation of educational software on urinary indwelling catheters. Rev Esc Enferm USP. [Internet]. 2011 [acesso em 15 fev 2018]; 45(1). Disponível em: http://dx.doi.org/10.1590/S0080-62342011000100030.

16. Pereira FGF, Caetano JA, Frota NM, Silva MG. Use of digital applications in the medicament calculation education for nursing. Invest Edu Enferm [Internet]. 2016 [acesso em 12 fev 2018); 34(2). Disponível em: http://dx.doi.org/10.17533/udea.iee.v34n2a09.

17. Pereira FGF, Silva DV, Sousa LMO, Frota NM. Building a digital application for teaching vital signs. Rev. Gaúcha Enferm. [Internet]. 2016 [acesso em 13 fev 2018]; 37(2). Disponível em: http:// dx.doi.org/10.1590/1983-1447.2016.02.59015.

Recebido: 10/03/2018

Finalizado: 28/01/2019

Autor Correspondente:

Francisco Gilberto Fernandes Pereira

Universidade Federal do Piauí

R. Cicero Eduardo S/N - 64600-000 - Picos, PI, Brasil

E-mail: gilberto.fp@hotmail.com

Contribuição dos autores:

Contribuições substanciais para a concepção ou desenho do estudo; ou a aquisição, análise ou interpretação de dados do estudo - DJLR

Elaboração e revisão crítica do conteúdo intelectual do estudo - GAAM, RMPLJ

Aprovação da versão final do estudo a ser publicado - FGFP, LMFF

Responsável por todos os aspectos do estudo, assegurando as questões de precisão ou integridade de qualquer parte do estudo - FGFP 